This item is the archived peer-reviewed author-version of:

\title{
3D camera assisted fully automated calibration of scanning laser Doppler vibrometers
}

\section{Reference:}

Sels Seppe, Ribbens Bart, Mertens Luc, Vanlanduit Steve.- 3D camera assisted fully automated calibration of scanning laser Doppler vibrometers

AIP conference proceedings / American Institute of Physics - ISBN 978-0-7354-1397-9 - 1740(2016), p. 090001-090009 Full text (Publishers DOI): http://dx.doi.org/doi:10.1063/1.4952688

To cite this reference: http://hdl.handle.net/10067/1347550151162165141 


\title{
3D Camera Assisted Fully Automated Calibration of Scanning Laser Doppler Vibrometers
}

\author{
Seppe Sels ${ }^{1, a), b)}$, Bart Ribbens ${ }^{1}$, Luc Mertens $^{1}$ and Steve Vanlanduit ${ }^{1}$ \\ ${ }^{1}$ Op3Mech Research Group, University of Antwerp, Salesianenlaan 90, 2660 Antwerp, Belgium

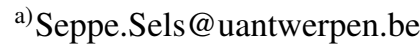 \\ b)URL: http://www.op3mech.com
}

\begin{abstract}
Scanning laser Doppler vibrometers (LDV) are used to measure full-field vibration shapes of products and structures. In most commercially available scanning laser Doppler vibrometer systems the user manually draws a grid of measurement locations on a $2 \mathrm{D}$ camera image of the product. The determination of the correct physical measurement locations can be a time consuming and difficult task.

In this paper we present a new methodology for product testing and quality control that integrates $3 \mathrm{D}$ imaging techniques with vibration measurements. This procedure allows to test prototypes in a shorter period because physical measurements locations will be located automatically.

The proposed methodology uses a 3D time-of-flight camera to measure the location and orientation of the test-object. The 3D image of the time-of-flight camera is then matched with the 3D-CAD model of the object in which measurement locations are pre-defined.

A time of flight camera operates strictly in the near infrared spectrum. To improve the signal to noise ratio in the time-of-flight measurement, a time-of-flight camera uses a band filter. As a result of this filter, the laser spot of most laser vibrometers is invisible in the time-of-flight image. Therefore a 2D RGB-camera is used to find the laser-spot of the vibrometer. The laser spot is matched to the 3D image obtained by the time-of-flight camera. Next an automatic calibration procedure is used to aim the laser at the (pre)defined locations.

Another benefit from this methodology is that it incorporates automatic mapping between a CAD model and the vibration measurements. This mapping can be used to visualize measurements directly on a 3D CAD model. Secondly the orientation of the CAD model is known with respect to the laser beam. This information can be used to find the direction of the measured vibration relatively to the surface of the object. With this direction, the vibration measurements can be compared more precisely with numerical experiments.
\end{abstract}

\section{INTRODUCTION}

The increasing availability of low cost and industrial 3D cameras has increased the research on 3D object recognition and pose estimation. Today object recognition and pose estimation are mainly used in robotics and 3D scanning techniques. In this paper we will combine 3D processing techniques with optical vibration measurement techniques. These optical vibration measurement techniques are frequently used in the design and testing of products and structures (e.g. automotive, machine or aircraft components)[1]. The scanning laser Doppler vibrometer (SLDV) is an accurate instrument based on laser interferometry that can measure high spatial resolution vibration data by sequentially positioning the measurement laser beam at discrete positions using two scanning mirrors (for the vertical and horizontal deflection of the beam). Commercial scanning laser Doppler vibrometers (see Figure 3) also have an integrated 2D RGB camera in which the position of the laser can be detected.

Although the scanning LDV is an accurate vibration measurement instrument, the measurement procedure involves a lot of user interaction: the user has to manually draw a grid of measurement locations on a 2D camera image of the measurement scene. Furthermore, the user also has to perform a calibration between the 2D image of the test object and the two laser mirror angles. This can be a time consuming and tedious task that has to be repeated every time that the instrument or the test object is moved.

Our proposed methodology uses an automated calibration procedure and 3D Time-of-Flight camera to measure the 
location and orientation of the test-object. The 3D image of the time of flight camera is then matched with the 3D CAD-model of the object in which measurement locations are pre-defined.

\section{STATE OF THE ART}

\section{Traditional Scanning LDV Techniques}

Currently the SLDV measurement procedure is time-consuming and it requires a lot of user input. When vibration shapes of an object are measured the user needs to go through the following steps:

1. Calibration of the scanning system: in the calibration step the transformation between the image coordinates $(x, y)$ and laser scanning angles $\left(\theta_{h o r}, \theta_{v e r}\right)$ are determined. To do this the user points the laser at minimum three positions on the structure and manually identifies these positions[2]. (see Figure 1a).

2. Definition of grid points on the object in the camera-image. In commercial software, polygon drawing tools are used for this purpose (see Figure 1b).

3. Measure the selected grid points by scanning over the defined grid points. (see Figure 1c).

During these steps, aiming the laser on a location on a structure is done by changing the angle of two integrated scanning mirrors. In order to be able to point at arbitrary locations on the structure during the measurement a mapping between the position of the laser spot and the angles of the mirrors is needed. This mapping is calculated with a 2D calibration procedure.

When the measurements are ready, they need to be interpreted and/or validated. With the traditional technique, measurements are only visualized on a standard 2D image of the object (see Figure 1c). Measurements are also difficult to compare with values from numerical simulations (e.g. finite element analysis) because no direct geometric correlation is available.

\section{Other Scanning LDV Methods}

Currently 3D and 2D localization techniques in combination with laser Doppler vibrometry are mainly used for measurements on moving objects. 2D techniques are used to track landmarks of a moving or rotating object and automatically aim the laser beam to a landmark position [3,4]. Although these methods are fast, the land-mark (white spot, reflective sticker) still needs to be applied manually. With these techniques 3D movements of the target where the landmark is distorted or only partially visible can cause inaccurate results. 3D techniques like photogrammetry are also used to track objects and aim the laser beam [5]. For accurate tracking manually applied or selected landmarks are still needed. None of the methods reported in literature provides a system that can automatically match measurements on a CAD-model and none of them can automatically select (visible) measurement points.

\section{Object Recognition and Matching}

Object recognition and matching of 3D CAD models with 3D point clouds are key parts of our methodology. Currently, 3D matching techniques exist and are implemented in open source libraries like PCL-C++ libraries [6]. In our work we mainly use viewpoint feature histograms [7] to detect an object in a point cloud and for finding an initial pose estimation. An implementation of the iterative closest point algorithm is used to refine the pose estimation [8,9].

\section{THE PROPOSES LDV SCANNING PROCEDURE}

\section{Methodology and Experimental Setup}

Our methodology eliminates all the manual user interaction of the traditional scanning technique. In our methodology the CAD model will be used to automatically select measurement points. These measurement points for the LDV measurements are inherent to a certain measurement object. The location of these points can thus be predefined on the CAD model of this object. Using pose estimation techniques we can locate this 3D CAD model in the 3D camera data. When the position of the object is known in camera coordinates, the positions of the measurement points are also 


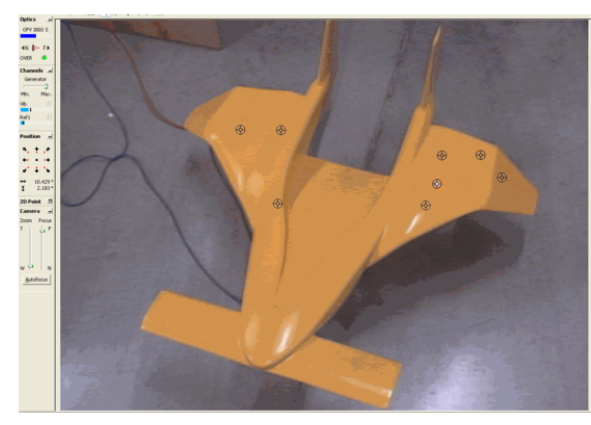

(a)

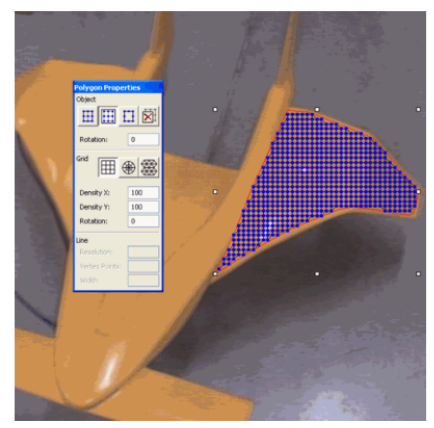

(b)

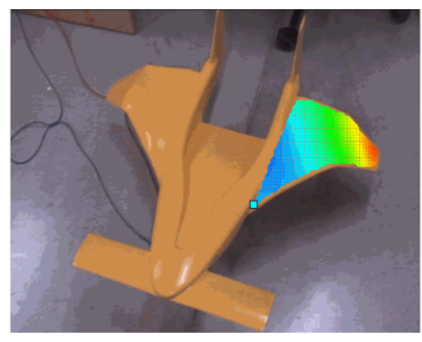

(c)

FIGURE 1: (a) Manual selection of calibration points. The 8 targets on the structure show the locations where the laser spot was placed and where the user clicked. (b) Manual selection of grid points superimposed on an RGB camera image in the Polytec scanning LDV system using a polygon selection tool. (c) Graphical representation of a vibration mode shape.

known. For aiming the laser with the scanning mirrors to these locations we use an automated calibration procedure. As a result of the complete methodology there is a direct correlation between the measurements and the CAD-model. Our proposed scanning methodology consists of the following steps:

1. Automatically calibrate the LDV scanning system (this is not object specific anymore)

2. Select measurement points on the CAD model (can be done beforehand)

3. Measure the vibrations at the predefined locations of interest.

The key role of the CAD model in our proposed methodology also allows for a more efficient measurement process. When simulations expose certain critical measurement points these points can be measured directly without measuring an entire grid around them. The link between all the proposed steps are visualized in Figure 2.

As 3D camera we use a Kinect One device. The device consists of a 3D Time-of-Flight (ToF) camera and a normal 2D RGB camera. The Kinect for windows SDK provides a pre-calibrated mapping between the 2D RGB coordinate system and the 3D Time-of-Flight system. The 3D TOF sensor is used for 3D measurements of the object. The measurements will be in the form of a 3D point cloud (see Figure 5). The ToF sensor is an active 3D sensor which operates in the near infrared spectrum. The LDV-laser operates in the visible spectrum (red light at $632 \mathrm{~nm}$ ). Because the ToF sensor is equipped with a band pass near infrared filter, the laser spot is not visible in the 3D image. Therefore, the integrated 2D RGB camera is used in the calibration procedure for detecting the laser spot. The Kinect is mounted below a scanning laser Doppler vibrometer (Polytec type psv 300). As test-object we used a model of a unmanned aerial vehicle that was developed by the company Nimbus in the framework of the FP7 Acheon ${ }^{1}$.

\section{Coordinate systems.}

The complete procedure uses different coordinate systems. In this section we provide an overview of the used coordinate systems and where they are used.

\footnotetext{
${ }^{1}$ http://acheon.eu/nimbus-develops-innovative-uav-configuration-for-the-acheon-experiments/ for more information.
} 


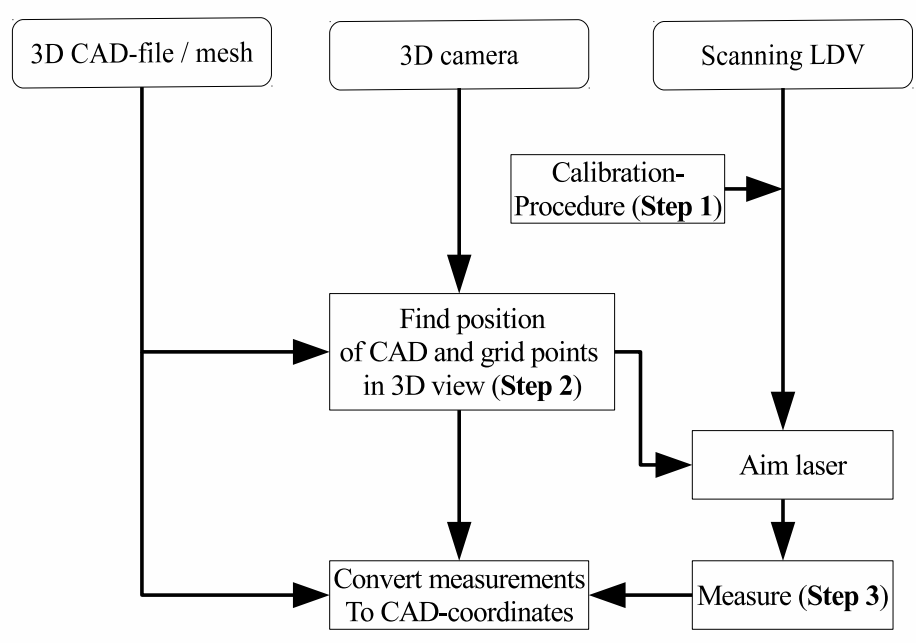

FIGURE 2: Link between measurements, CAD-model, 3D camera and scanning LDV. A 3D camera, 3D model and pose estimation is used to find the position of the object and automatically select measurement points. An automated calibration procedure is used to aim the laser to these points.

- Calibration

2D coordinate system $(x, y)_{r g b}$ of RGB-camera

LDV scanning mirror-angles $\left(\theta_{\text {hor }}, \theta_{\text {ver }}\right)$

- $\quad 3 \mathrm{D}$ measurements

3D coordinate system $(x, y, z)_{3 D}$ of 3D-camera

- $\quad$ CAD model or mesh model

3D coordinate system $(x, y, z)_{C A D}$ of 3D model in CAD-file

- LDV-laser position

Defined by mirror-angles $\left(\theta_{h o r}, \theta_{v e r}\right)$. Converted to 2D coordinate system of RGB-camera $(x, y)_{r g b}$.

- Sample positions (grid)

Is used in multiple coordinate systems

2D coordinate system of RGB camera $(x, y)_{r g b}$

3D coordinate system of 3D camera $(x, y, z)_{3 D}$

3D coordinate system of CAD-model $(x, y, z)_{C A D}$

- LDV-measurement

1D coordinate system (direction of laser)

In our procedure converted to $3 \mathrm{D}$ coordinate system of the CAD-model

\section{Proposed Calibration Procedure}

The new calibration procedure uses the 2D coordinate system of an RGB camera[2] like traditional techniques but is fully automatic. The calibration-procedure consists of the following steps:

1. Position the scanning LDV-laser at random locations within the 2D camera-view (e.g. 100 locations)

2. Find laserspot $x y$-coordinates in (RGB) image at different mirror-angles

3. Calculate mapping between $(x, y)_{r g b}$ coordinates and scanning mirror angles $\left(\theta_{h o r}, \theta_{v e r}\right)$ [2]

4. Calculate mapping between $(x, y)_{r g b}$ coordinates and scanning mirror angles $\left(\theta_{\text {hor }}, \theta_{v e r}\right)$ [2]

The calibration calculates $d$ and $d_{0}$ from Equation 2 and Equation 3 with singular value decomposition[2]:

$$
\begin{gathered}
x=d \tan \left(\theta_{h o r}\right) \\
y=d_{0}+\frac{d \tan \left(\theta_{v e r}\right)}{\cos \left(\theta_{h o r}\right)}
\end{gathered}
$$




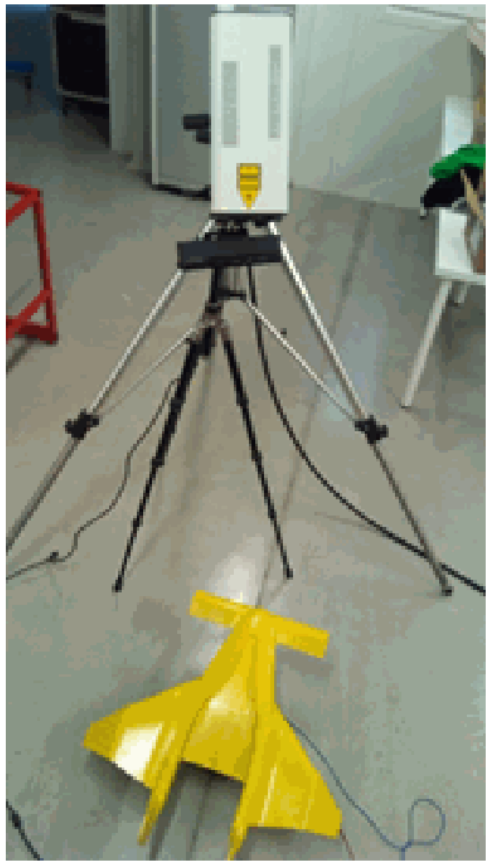

(a)

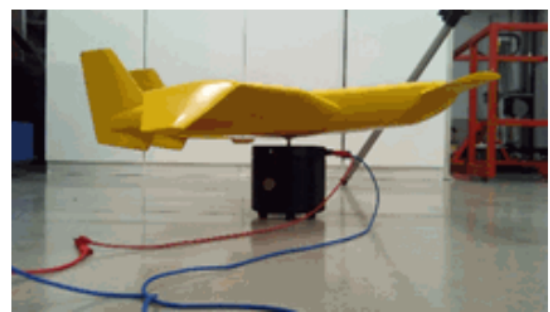

(b)

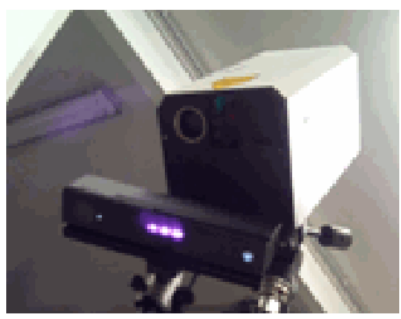

(c)

FIGURE 3: Example of experiment setup. Kinect One 3D camera mounted below a Polytec psv 300 scanning LDV aimed to the sample-object (a). Sample object mounted on a electromagnetic shaker (c) Kinect One 3D camera and front of scanning LDV.

The laser spot is detected by background subtraction of a reference frame (where no laser-spot is seen) and a frame where the lasers pot is visible. The highest values of this differentiated image will indicate the position of the laser spot.

\section{Automatic Grid Selection}

To automatically select grid points for scanning an object different steps are taken:

1. Find the object position: Find the coordinate transformation between the CAD-model and the object seen in the measured 3D point cloud.

2. Detect which grid-points are visible for the scanning LDV.

These two steps are explained below. A complete summary is given in Figure 7.

To find the CAD-model in a complete 3D scene, a training set of different views of the model is built. To generate this training set a virtual model of a 3D camera is used. From different poses a virtual 3D view (point cloud) is made of the model. From this point cloud a global viewpoint feature histogram (VFH)[7] is calculated. This histogram consists of bins that describe different features of the object. These features are dependent of the pose of the object (viewpoint) and shape-features (surface-shape, edges,...) of the object. This histogram is later used to find the object in the measured point cloud and also provides an initial pose estimation of the object.

In the following step the position of an object in camera-coordinates is obtained by matching a 3D-model (CADfile) and the measured 3D point cloud. This matching consists of the following steps:

1. Cluster Cloud (Euclidian clustering) + Calculate VFH's for each cluster.

2. Find closest match in training set (cosine similarity distance).

3. If distance between cluster and trainingset is small: the cluster is the object

4. If the object is found, use the closest match of the training set as an initial pose estimation 


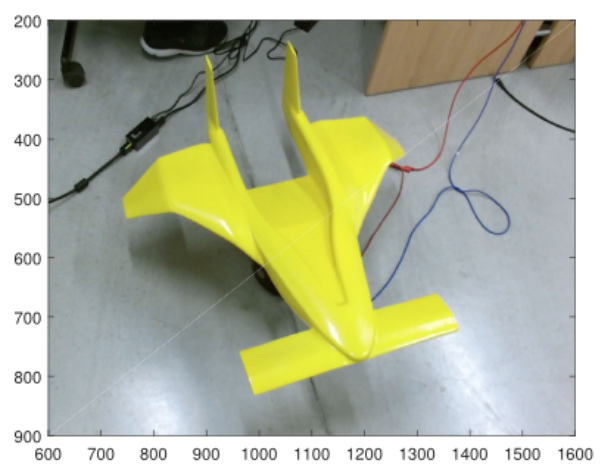

(a)

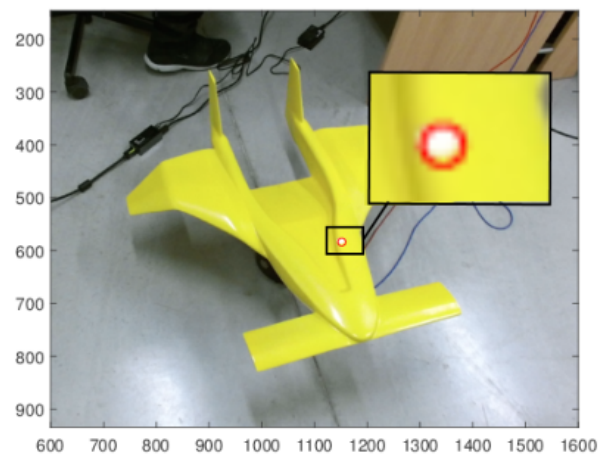

(c)

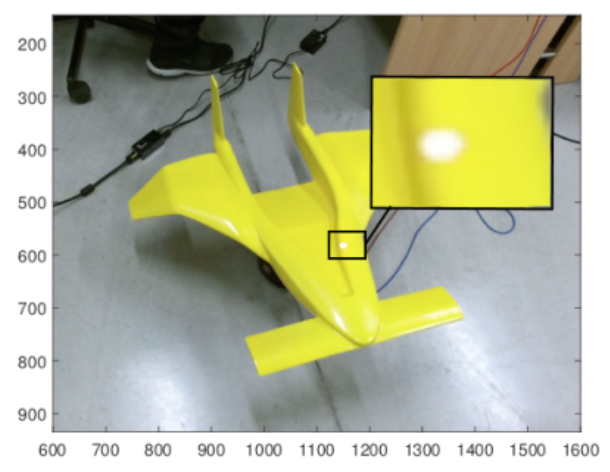

(b)

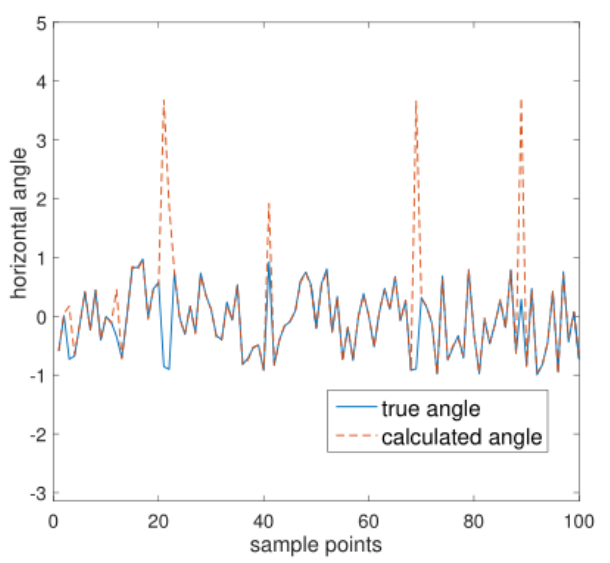

(d)

FIGURE 4: Used images in calibration procedure. (a) Reference image without laser spot. (b) Image with laserspot. (c) Detected laser spot by detecting difference between reference image and new image.(d) Comparison between true (applied) angle and calculated angles (only horizontal angles are shown). Some outliers exist due to bad detected sample points.

5. Use the Iterative Closest points (ICP) $[8,9]$ algorithm to refine the transformation matrix between the CAD-file and measured point cloud.

All used algorithms (VFH, ICP) use functions implemented in the pointcloud-library (PCL) [6, 7] .

When the position of the model is known relative to the 3D camera we can also transform points of interest (measuring/grid points) to the 3D camera coordinate system. These points can be predefined in the 3D-CAD model, or a mesh representation of the CAD-model can be used. The points of interest are projected to a virtual representation of the 3D sensor. If the point of interest is visible in the virtual image it is considered visible in the real world by the SLDV. The visible points of interest are the grid-points that will be measured by the SLDV. The grid-points 3D coordinates are converted to (see calibration procedure) mirror angles. These LDV mirrors will point the laser towards a grid point. The procedure is summarized in the following steps:

1. Use the calculated transformation between the CAD reference frame and a 3D point cloud frame to transform the desired measurement point to the 3D point cloud scene.

2. Use a virtual model of the $3 \mathrm{D}$ camera to find visible measurement points. 


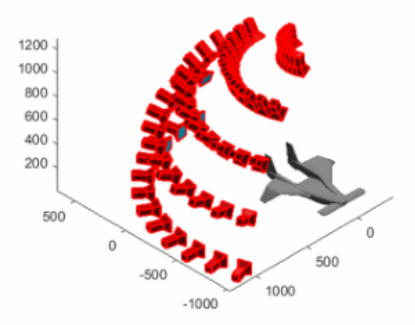

(a)

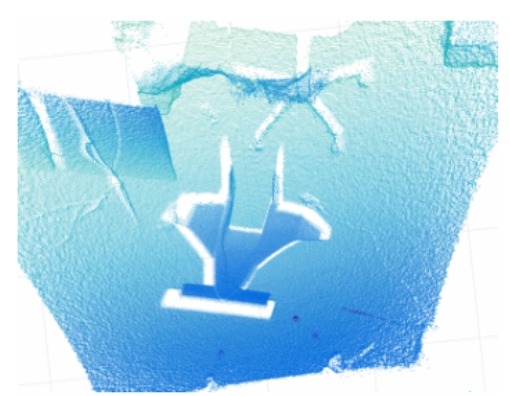

(c)

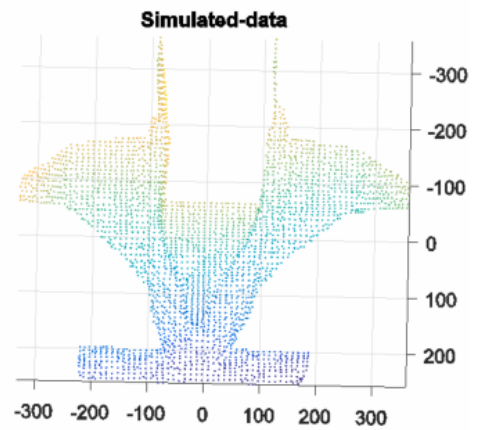

(b)

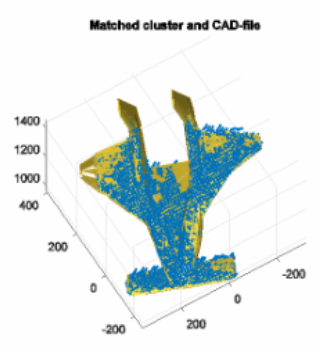

(d)

FIGURE 5: (a) Partial (1/4) set of training-views. Virtual Cameras are located in a sphere around the CAD object. The generated point clouds, VFHs and transformation-matrices are saved. (b) Example synthetic 3D view. (c) Measured point cloud. (d) Matched cluster and CAD file.

3. Transform 3D coordinates to $2 \mathrm{D}-\mathrm{RGB}$ pixel coordinates

4. Use the proposed calibration method to map the RGB pixel coordinate to LDV mirror angles.

5. Measure the vibrations in the chosen point and link the obtained measurement to the $3 \mathrm{D}$ coordinate of the point in the CAD reference frame.

Below we describe the transformations (homogenous coordinates) to convert a point in CAD coordinates to the scanning mirror angles of the LDV system.

$$
\begin{gathered}
P(x, y, z, 1)_{3 D}=T_{c a d \rightarrow 3 D} \cdot P(x, y, z, 1)_{c a d} \\
P(x, y, 0,1)_{r g b}=T_{3 D \rightarrow r g b} \cdot P(x, y, z, 1)_{p c} \\
P\left(\theta_{\text {hor }}, \theta_{v e r}\right)=T_{r g b \rightarrow \theta} \cdot P(x, y, 0,1)_{r g b}(\text { see eq. } 1 \text { and } 2)
\end{gathered}
$$

1. Cluster Cloud (Euclidian clustering) + Calculate VFH's for each cluster.

2. Find closest match in training set (cosine similarity distance).

3. If distance between cluster and trainingset is small: the cluster is the object

4. If the object is found, use the closest match of the training set as an initial pose estimation

5. Use the Iterative Closest points (ICP) $[8,9]$ algorithm to refine the transformation matrix between the CAD-file and measured point cloud.

All used algorithms (VFH, ICP) uses functions implemented in the pointcloud-library (PCL) [6, 7] . 


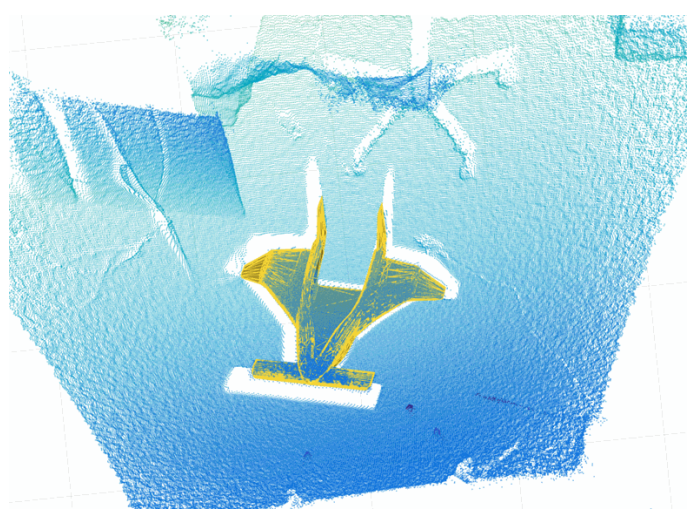

(a)

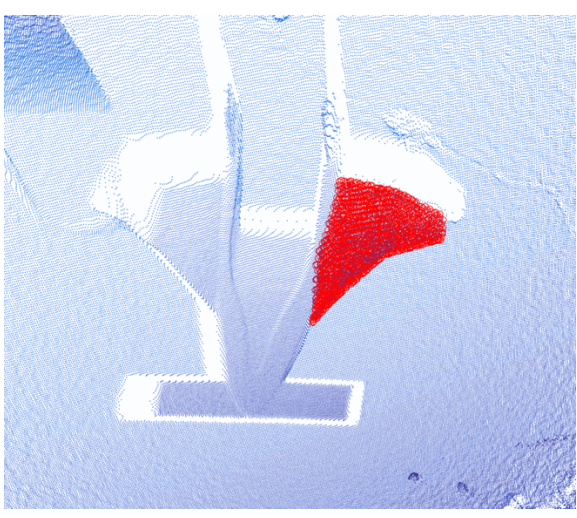

(b)

FIGURE 6: (a) Matched CAD-file (yellow/lines) and point cloud. (b)Mapped mesh/measurement (red) for scanning the right wing of the drone

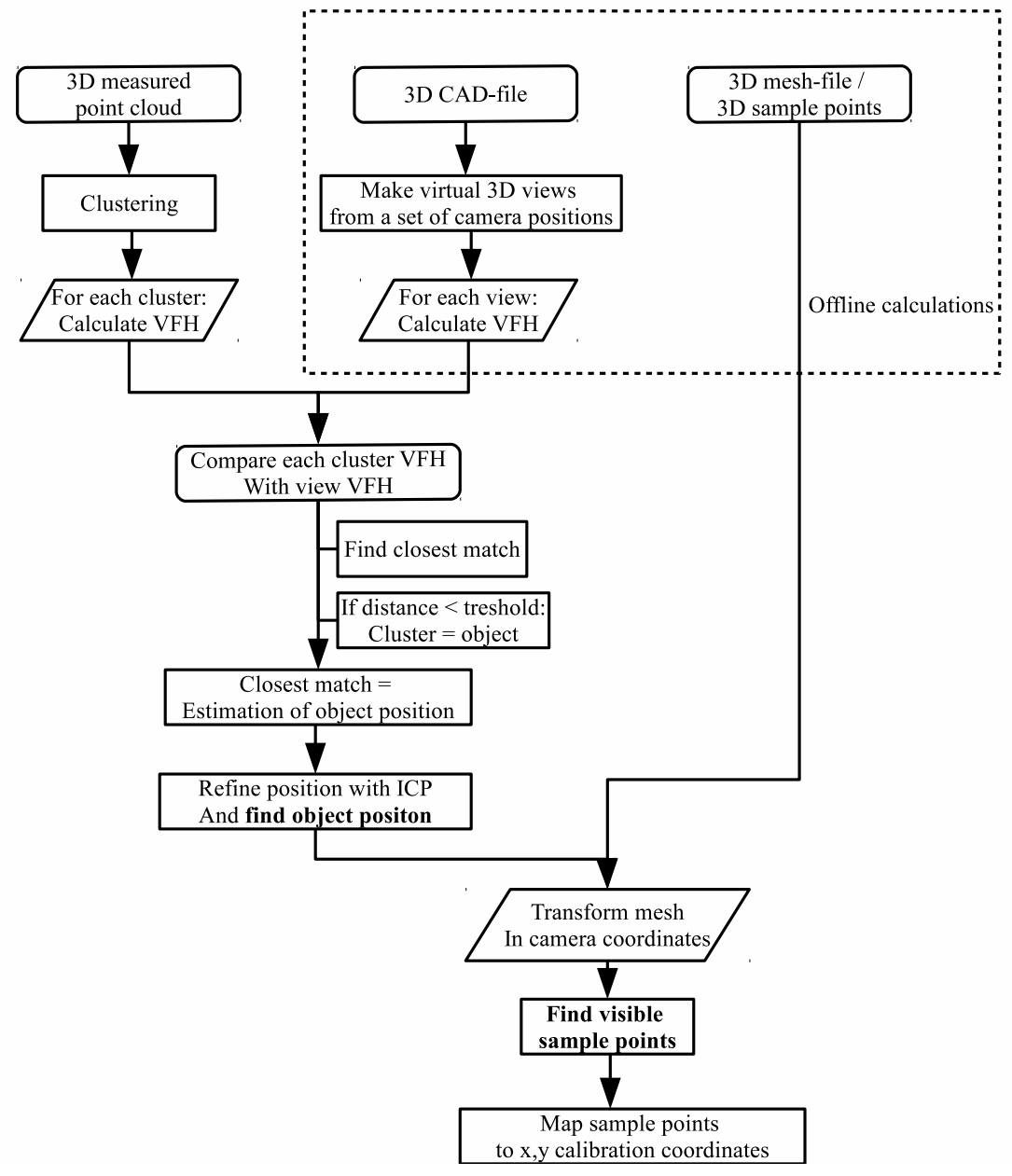

FIGURE 7: Flow-chart relating the different aspects of the proposed method to the global process. 


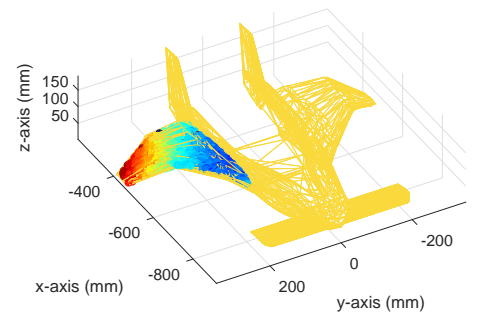

(a)

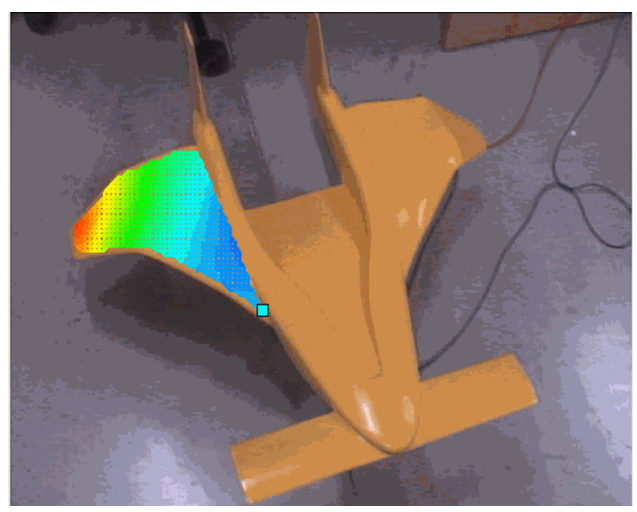

(b)

FIGURE 8: Comparison between (a) our new 3D method and (b) the original output from Polytec. Note that the polytec viewer filters (smooths) the output where our visualization shows the real (unfiltered) results.

\section{RESULTS}

With our methodology it is possible to correlate the LDV measurements directly with the CAD model. As a result it is easier to compare real measurements with numeric simulations. In Figure 8 we compare the result of a traditional scanning method (Polytec) with the result of the proposed procedure. The measurements are mapped on a 2D view of the object. In this view it is difficult to get the exact position of the scanning grid. And therefore it is difficult to compare to numeric simulations.

\section{CONCLUSION}

Scanning laser Doppler vibrometers (SLDV) are accurate instruments based on laser interferometry that can measure high spatial resolution vibration data. Current techniques require a lot of user interaction during the calibration of the SLDV-system and setup of the measurement. Our methodology eliminates this user interaction with an automated calibration procedure and the detection of the test-object using a the 3D model of the test-subject and a 3D camera. The methodology also incorporates automatic mapping between a CAD model and the vibration measurements. This mapping is used to directly visualize measurements on a 3D CAD model. Also this mapping can be used to directly compare measurements and numeric simulations.

\section{REFERENCES}

[1] C. Pickering, N. Halliwell, and T. Wilmshurst, Journal of Sound and Vibration 107, $471-485$ (1986).

[2] S. Vanlanduit, P. Guillaume, B. Cauberghe, and P. Verboven, Measurement Science and Technology 14, p. 1469 (2003).

[3] P. Castellini and E. Cupido, Proceedings of the International Modal Analysis Conference IMAC 1, 457-463 (2001).

[4] P. Castellini, Shock and Vibration 9, 67-89 (2002).

[5] a. Jennings, J. Black, C. Allen, J. Simpkins, and R. Sollars, Experimental Mechanics 51, 71-84 (2011).

[6] PCL, "pointclouds.org @ONLINE," November (2015).

[7] R. B. Rusu, G. Bradski, R. Thibaux, and J. Hsu, "Fast 3d recognition and pose using the viewpoint feature histogram," in Intelligent Robots and Systems (IROS), 2010 IEEE/RSJ International Conference on (2010), pp. 2155-2162.

[8] P. Bergstrm, "Computational methods for on-line shape inspection," Ph.D. thesis 2009.

[9] B. Bellekens, V. Spruyt, R. B. M. Weyn, and R. Berkvens, The Fourth International Conference on Ambient Computing, Applications, Services and Technologies (2014). 